American Journal of Computer Engineering
(AJCE)

\title{
Underwater Wireless Sensor Networks Challenges: A Review
}

\section{Taranpreet Kaur}

Assistant Professor, Dept: Computer Science, Mata Gujri College, Sri Fatehgarh Sahib

\begin{abstract}
The underwater wireless sensor network consists of sensors that ${ }^{*}$ Correspondence to Author: enable the exploration of natural undersea resources and gather- Taranpreet Kaur ing scientific data in collaborative monitoring missions. Underwa- Assistant Professor ter wireless sensor Network consists of variable number of float- Dept: Computer Science, Mata Guing and anchored sensors, sink and vehicles that are deployed jri College, Sri Fatehgarh Sahib over an area to be explored. Applications of underwater sensing range from oil industry to aquaculture, and include instrument monitoring, pollution control, climate recording, Prediction of natural disturbances, search and survey missions, and study of How to cite this article:

marine life. UWSN is a network of autonomous sensor nodes. Taranpreet Kaur.Underwater WireWhich are spatially distributed underwater to sense the water-re- less Sensor Networks Challenges: lated properties such as quality, temperature, and pressure? The A Review. American Journal of sensed data can be utilized by variety of applications that can Computer Engineering, 2018; 1:2. be used for the benefit of humans. The sensor nodes, stationary or mobile are connected wirelessly via communication modules to transfer various events of interest. In this paper, several fundamental key aspects of underwater acoustic communications are investigated. Different architectures for two-dimensional and three-dimensional underwater sensor networks are discussed, and the underwater channel is characterized. The main challeng- eSciPub LLC, Houston, TX USA. es for the development of efficient networking solutions posed Website: http://escipub.com/ by the underwater environment are detailed at all layers of the protocol stack. Furthermore, open research issues are discussed and possible solution approaches are outlined.

Keywords: Underwater Wireless Sensor Networks, Applications and Challenges
\end{abstract}




\section{Introduction:}

Today, both the vehicle technology and the sensor technology are mature enough to motivate the idea of underwater sensor networks. To turn this idea into reality, however, one must face the problem of communications. Underwater communication systems today mostly use acoustic technology. Complementary communication techniques, such as optical and radio-frequency, or even electrostatic communication, have been proposed for short-range links (typically 1$10 \mathrm{~m})$, where their very high bandwidth $(\mathrm{MHz}$ or more) can be exploited. These signals attenuate very rapidly, within a few metres (radio) or tens of metres (optical), requiring either high-power or large antennas. Acoustic communications offer longer ranges but are constrained by three factors: limited and distance-dependent bandwidth, time-varying multi-path propagation and low speed of sound. Together, these constraints result in a communication channel of poor quality and high latency, thus combining the worst aspects of terrestrial mobile and satellite radio channels into a communication medium of extreme difficulty. Among the first underwater acoustic systems was the submarine communication system developed in the USA around the end of the Second World War. It used analogue modulation in the $8-11 \mathrm{kHz}$ band (singlesideband amplitude modulation). Research has since advanced, pushing digital modulationdetection techniques into the forefront of modern acoustic communications. At present, several types of acoustic modems are available commercially, typically offering up to a few kilobits per second (kbps) over distances up to a few kilometres. Considerably higher bit rates have been demonstrated, but these results are still in the domain of experimental research. The $85 \%$ of earth's surface is covered with water in the form of rivers, canals, seas, and oceans. Plenty of precious resources lie underwater which are required to be explored. To know about underwater applications, there are some design challenges and tools from ground based wireless sensor networks. Even underwater networks resemble like terrestrial networks, there are some fundamentally different from terrestrial networks which are not suitable for the underwater environment. Radio signals in terrestrial networks are not applicable for underwater networks due to the propagation of long distances at very low frequencies, requires high transmission power and large antennas. Due to the shadow zones, temporary connectivity loss is experienced. When compared with radio waves; acoustic waves have high propagation delay, low or insufficient bandwidth, path loss and more consumption of energy. Additionally, some other challenges such as continuous movement of sensor nodes, which is unsuitable for the (GPS) Global Positioning System for the underwater environment.

\section{Under Water Sensing Application:}

Applications can have very different requirements: fixed or mobile, short or longlived, best-effort or life-or-death; these requirements can result in different designs. In next describe different kinds of deployments, classes of applications and several specific examples, both current and speculative.

\section{Deployments:}

Deployment of the underwater sensor networks, Mobility and density are two parameters that vary over different types of deployments of underwater sensor networks. According to the advanced D-S theory of evidence, the change of reliability coverage in underwater sensor networks' detection area was analyzed, and a Nodes-deployment Algorithm Based on Advanced D-S Theory of Evidence and Prior Probability (NAAEP) combining with the prior probability of target nodes distribution was proposed. The value of the target perceived reliability was transformed into a virtual potential field force that a node was suffered in size, and the position of the nodes was adjusted according to the force that nodes were suffered in potential field. 
Simulation model of underwater sensor networks was established using MATLAB. The simulation results show the effectiveness of the algorithm, energy consumption and the number of deployment nodes is reduced, and the scope of perceiving is expanded. Here, we focus on wireless underwater networks, although there is significant work in cabled underwater observatories, from the sound surveillance system military networks in the 1950s, to the recent Ocean Observatories Initiative. As with surface sensor networks, network density, coverage and number of nodes are interrelated parameters that characterize a deployment. Underwater deployments to date are generally less dense, have longer range and employ significantly fewer nodes than terrestrial sensor networks. For example, the Seaweb deployment in 2000 involved 17 nodes spread over a $16 \mathrm{~km} 2$ area, with a median of five neighbours per node. Finally, as with remote terrestrial networks, connectivity to the Internet is important and can be difficult. Figure shows several options, including underwater cables, point-to-point wireless and satellite.

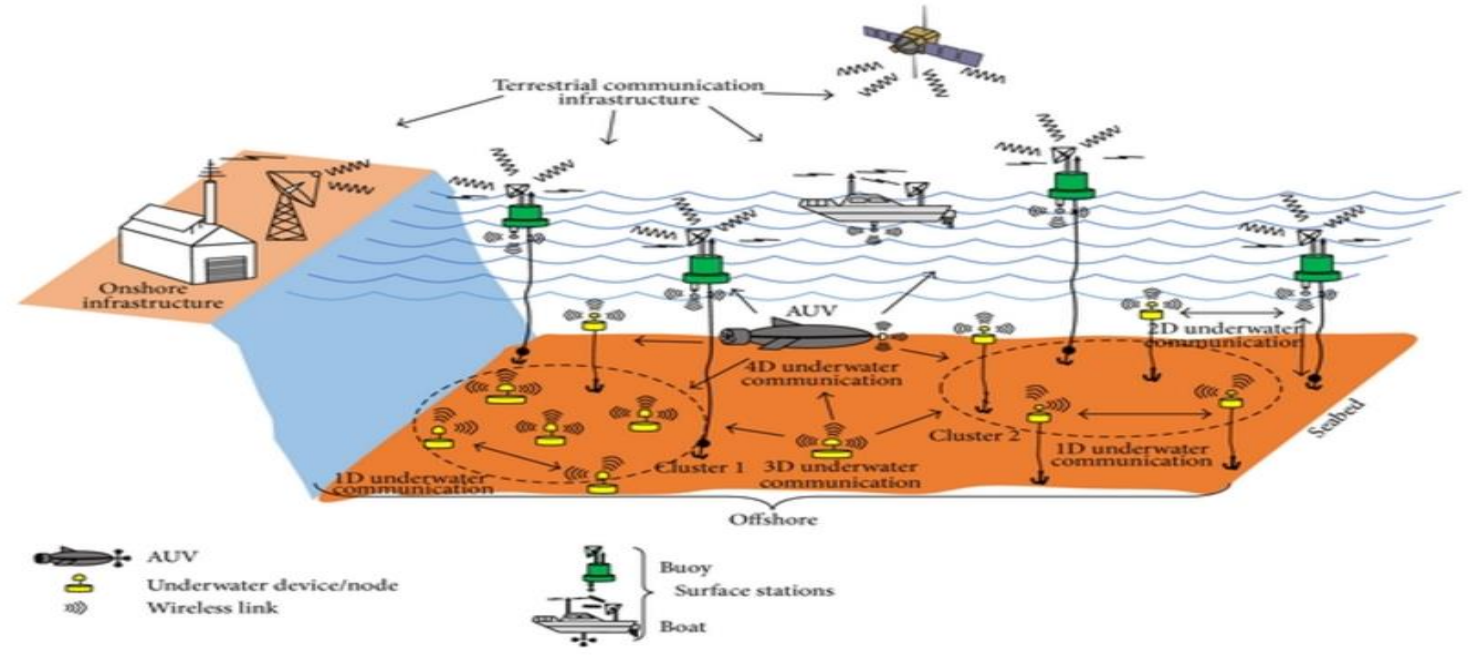

\section{Application domains:}

Applications of domain underwater networks fall into three categories:

Scientific applications observe the environment: from geological processes on the ocean floor, to water characteristics (temperature, salinity, oxygen levels, bacterial and other pollutant content, dissolved matter, etc.) to counting or imaging animal life (micro-organisms, fish or mammals).

Industrial applications monitor and control commercial activities, such as underwater equipment related to oil or mineral extraction, underwater pipelines or commercial fisheries. Industrial applications often involve control and actuation components as well. Military and homeland security applications involve securing or monitoring port facilities or ships in foreign harbours, de-mining and communication with submarines and divers.

\section{Underwater communications}

Underwater wireless communication refers to transmitting data in unguided water environment through the use of wireless carriers, i.e., radio-frequency wave, acoustic wave, and optical wave. The underwater wireless optical communication employs optical wave as the transmission carriers. In comparison to RF and acoustic counterparts, has a much higher transmission bandwidth, thus providing much higher data rate. Due to this high-speed transmission advantage, underwater communications has attracted considerable attention in recent years. Many 
potential applications of underwater communication systems have been proposed for environmental monitoring, offshore exploration, disaster precaution, and military operations

Major challenges in the design of underwater sensor network are:

- Battery power is limited and usually batteries cannot be recharged, also because solar energy cannot be exploited;

- The available bandwidth is severely limited;

- Channel characteristics, including long and variable propagation delays, multipath and fading problems;

- High bit error rates;

- Underwater sensors are prone to failures because of fouling, corrosion, etc.

\section{Networking technology:}

A number of hardware platforms for acoustic communication have been developed over the years, with commercial, military and research success. These platforms are essential to support testing and field use. The Teledyne/Benthos modems are widely used commercial devices. They have been extensively used in Sea Web, with vendorsupported modifications, but their firmware is not accessible to general users, limiting their use for new physical layer and MAC research. The Ecologic S2C modems may provide some additional flexibility in that they support the transmission of short packets, which are completely customizable by the users and can be transmitted instantly without any medium access protocol rule (this feature is also supported by the WHOI micro-modem). By using such packets, there is some room for implementing and testing protocols, even though the level of reprogram ability of commercial devices remains rather limited in general. The data rates supported by these modems range from a few hundred bps to a few kbps in various bands of the tens of $\mathrm{kHz}$ frequency range, over distances up to a few tens of kilometers and with power consumptions of tens of watts.

Some types of underwater sensors are easy and inexpensive, but many rapidly become difficult and expensive-from a few dollars to thousands or more. Inexpensive sensors include pressure sensing, which can give approximate depth, and photo-diodes and thermostats that measure ambient light and temperature. More specialized sensors include flourometers that estimate concentrations of chlorophyll, and devices to measure water $\mathrm{CO} 2$ concentrations or turbidity, and sonar to detect objects underwater. Such specialized sensors can be much more expensive than more basic sensors. Traditional biology and oceanography rely on samples that are taken in the environment and returned to the laboratory for analysis. As traditional underwater research has assumed personnel on site, the cost of sample return is relatively small compared with the cost of getting the scientist to the site. With lower cost sensor networks and AUVs, we expect the costs of sample-return relative to sensing to force revisiting these assumptions. Sonar has been used for over more than 60 years for processing single sensors and sensor-array data, and today, offline, premission planning of AUVs has become routine. As the field matures, we look forward to work involving online, adaptive sampling using communicating AUVs.

\section{Conclusion:}

While research on underwater sensor networks has significantly advanced in recent years, it is clear that a number of challenges still remain to be solved. With the new approaches to communication, medium access, networking and applications, effective analysis, integration and testing of these ideas is paramount-the field must develop fundamental insights, as well as understand what stands up in practice. For these reasons, we believe that the development of new theoretical models (both analytical and computational) is very much needed, and that 
greater use of in the field experiments; such work will support more accurate performance analysis and system characterization, which will feed into the next generation of underwater communications and sensing. In addition, integration and testing of current ideas will stress the seams that are often hidden in more focused laboratory research, such as total system cost, energy requirements and overall robustness in different conditions. While the vast amount of solutions for terrestrial WSNs provides a valuable insight into networking in this environment, there exist many challenges unique to underwater communication. Especially, the significantly different characteristics of communication in water require many networking paradigms to be revisited.

\section{REFERENCES:}

1. A Survey of Underwater Wireless Optical Communication by Zhaoquan ZengB.Eng., Tianjin University, P. R. China, 2013

2. Study of Applications of Underwater Wireless Sensor Networks M. Kiranmayi1, Dr. Kathirvel Ayyaswamy2

3. M. Stojanovic, "Acoustic (underwater) communications," in Encyclopedia of Telecommunications, J. G. Proakis, Ed. John Wiley and Sons, 2003.

4. P. Xie, J.-H. Cui, and L. Lao, "VBF: vector-based forwarding protocol for underwater sensor networks," Networking, vol. 3976, pp. 1216-1221, 2006.

5. The Challenges of Building Scalable Mobile Underwater Wireless Sensor Networks for Aquatic Applications, Jun-Hong Cui, University of Connecticut, Storrs Jiejun Kong and Mario Gerla, University of California at Los Angeles Shengli Zhou, University of Connecticut, Storrs.

6. Underwater Sensor Network Applications: A Comprehensive SurveyEmadFelemban, FaisalKarimShaikh, UmairMujtabaQureshi, AdilA.Sheikh, andSaadBinQaisar, International Journal of Distributed Sensor Networks Volume 2015.

7. Underwater sensor networks: applications, advances and challenges BY JOHN HEIDEMANN $1,{ }^{*}$, MILICA STOJANOVIC2 AND MICHELE ZORZI3 1Information Sciences Institute, University of Southern California, Marina del Rey, CA, USA, on March 21, 2018.

8. Challenges for Efficient Communication in Underwater Acoustic Sensor Networks lan F. Akyildiz, Dario Pompili, Tommaso Melodia,Broadband \& Wireless Networking Laboratory School of Electrical \& Computer Engineering Georgia Institute of Technology, Atlanta, GA.

9. M. Stojanovic, "Acoustic (underwater) communications," in Encyclopaedia of Telecommunications, J. G. Proakis, Ed. John Wiley and Sons, 2003.

10. J. Proakis, J. Rice, E. Sozer, and M. Stojanovic, "Shallow water acoustic networks," in Encyclopedia of Telecommunications, J. G. Proakis, Ed. John Wiley and Sons, 2003.

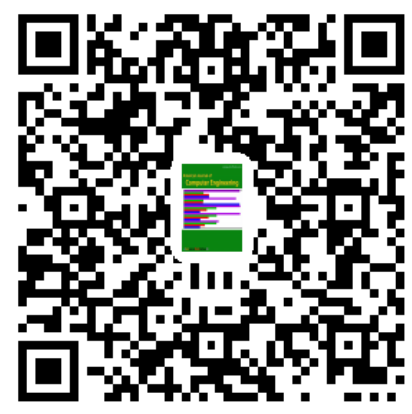

